\title{
DEMENCIA FRONTOTEMPORAL NO FAMILIAR Y EPILEPSIA GENERALIZADA
}

\author{
Patricio Fuentes, Andrea Slachevsky, Pablo Reyes, Luis Cartier
}

\begin{abstract}
RESUMEN - Se presenta un paciente de 62 años, sin antecedentes familiares de demencia, que a los 45 años debuta con crisis súbitas de disnea, visión borrosa, caída al suelo y movimientos repetitivos de brazos. Tratado por epilepsia con fenitoína y ácido valproico, repite esporádicamente crisis semejantes. Hace 4 años sus familiares notan cambios de personalidad, irritabilidad y conductas obsesivas. Hace 2 años aparecen episodios de desorientación de días de duración, algunos con alucinaciones auditivas y también fenómenos convulsivos. Ultimamente presenta crisis polimorfas, algunas con prolongada alteración de conciencia, estados catatoniformes y relajación esfinteriana. Examen físico y neurológico sin anormalidades. Evaluación neuropsicológica evidenció consistentes defectos en funciones frontales. EEG mostraron lentitud generalizada y actividad irritativa esporádica en regiones frontotemporales. Atrofia cortical de predominio anterior en CT scan e hipoperfusión fronto-temporal bilateral en SPECT. Exámenes de laboratorio y LCR normales. Conclusión: La asociación de DFT con epilepsia, en forma no familiar, sugiere un síndrome neurodegenerativo cortical diferente.
\end{abstract}

PALABRAS-CLAVE: demencia frontotemporal, epilepsia generalizada, síndrome catatónico.

\section{Frontotemporal dementia non familial and generalized epilepsy}

\begin{abstract}
A 62 year-old patient is presented, without family antecedents of dementia who begins with 45 years of age with sudden crisis of dyspnea, blurred vision, fall to the floor and repetitive jerks of arms. Tried by epilepsy with phenytoin and valproate repeats similar crisis sporadically. Four years ago their re latives began to notice changes of personality, irritability and obsessive behaviors. Later on, are added episodes of disorientation of days of duration, some with auditory hallucinations and also convulsive manifestations. Finally appear polymorphic crisis, some with continue alteration of consciousness, catatonic states and sphincteric incontinence. Physical and neurological examination without abnormalities. Neuropsychological evaluation evidenced consistent defects in frontal functions. EEG showed widespread slowness and sporadic irritative activity in frontotemporal regions. Anterior cortical atrophy in CTscan and bilateral frontotemporal hypoperfusion in SPECT. Labs exams and CSF were nomal. Conclusion: The association of FTD and epilepsy, in non-family form, suggests a different neurodegenerative cortical syndrome.
\end{abstract}

KEY WORDS: frontotemporal dementia, generalized epilepsy, catatonic syndrome.

La demencia frontotemporal (DFT) es la expresión clínica de la degeneración frontotemporal, junto con la demencia semántica y la afasia pro$\mathrm{g}$ resiva no fluente. Esta forma de demencia, cuyo criterio diagnóstico exige la combinación de un desorden conductual y un síndrome disejecutivo progresivo, es la segunda causa más frecuente de demencia degenerativa en individuos menores de 65 años'. Una historia familiar de demencia está presente en aproximadamente la mitad de los pacientes y el $10 \%$ de los casos familiares han sido relacionados con mutaciones específicas del gen de la proteína tau, ubicado en el cromosoma $17^{2}$. Algunas alteraciones neurológicas han sido iden- tificadas en pacientes con DFT, amiotrofia en el contexto del desarrollo de una enfermedad de moto ne u rona, también se ha descrito parkinsonismo (aquinesia, rigidez y temblor) en fases tardías de la enfermedad) y la notable presencia de reflejos primitivos como el de succión y de prehensión ${ }^{3}$.

Se describe un paciente de 62 años, sin antecedentes familiares de demencias, quien presenta ep $\mathrm{i}-$ lepsia de aparición tardía, con crisis de variada expresión y que años más tarde desarrolla una demencia de perfil frontotemporal. Se discute la posibilidad de una asociación sindromática degenerativa no descrita ó la concurrencia de dos entidades secuenciales independientes.

Servicio de Neurología, Hospital del Salvador, Santiago, Chile.

Recebido 22 Março 2005. Aceito 7 Julho 2005.

Dr. Patricio Fuentes - Avenida Salvador 364 - Santiago Chile -E-mail: pfuentes@mi.cl 


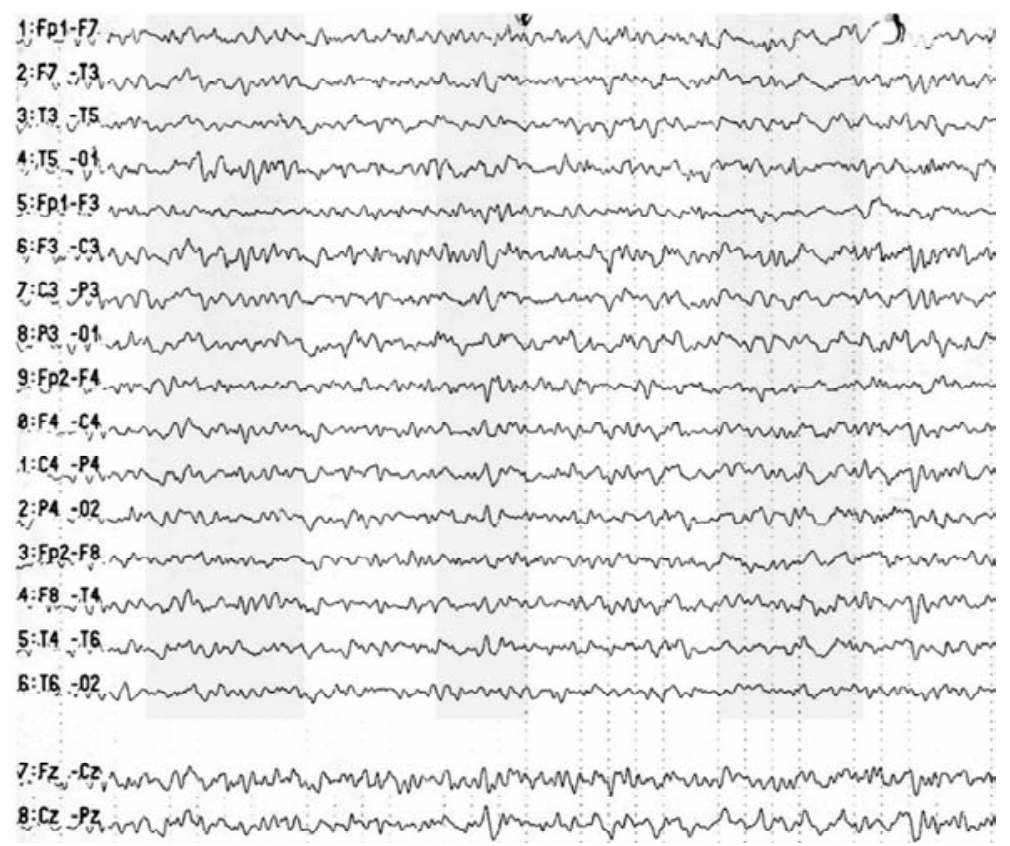

Fig 1. Paroxismos lentos de mayor ampli tud frontotemporal izquierda sobre fondo theta de bajo voltaje difuso y simétrico.

\section{CASO}

Hombre de 62 años, sin antecedentes hereditarios de epilepsia o demencia, que en 1987 debuta con crisis de disnea, visión borrosa, caída al suelo, movimientos repetitivos de los brazos y estado confusional de duración total de 10 minutos. Fue tratado con fenitoína y ácido valproico con lo que permaneció estable, presentando sólo 3 años después nueva crisis con similares características. En Abril del año 2003, la cónyuge comienza a notar cambios conductuales, desinterés, irritabilidad y comportamiento obsesivo.

Se hospitaliza en Octubre del año 2003 por cuadro de compromiso de conciencia pro g resivo de varios días de evolución, agitación psicomotora, desorientación, desviación de la mirada hacia arriba y derecha, temblor del mentón y parpadeo. Al ingreso se encontró además distonías de las extremidades y mantención espontánea

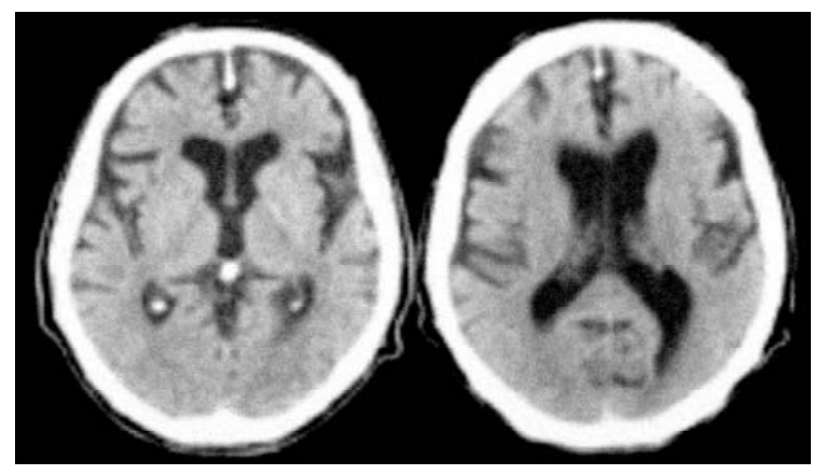

Fig 2. Tomografia axial computada de encéfalo que evidencia una gran atrofia frontotemporal. de posturas de apariencia catatónica, fenómenos que cedieron con el uso de diazepam endovenoso. Durante esta hospitalización presentó varios otros episodios catatoniformes que duraban horas o días y que en algunas oportunidades se asociaron a mioclonías faciales y de la mano derecha. EI EEG se mantuvo persistentemente alterado con actividad lenta generalizada (Fig 1). Se interpretó clínicamente como un cuadro epiléptico, con buena respuesta a los anticonvulsivantes. El estudio imagenológico mostró una atrofia frontal bilateral. El estudio de laboratorio obtuvo una amonemia alta (44 $\mu \mathrm{mol} / \mathrm{L})$ que fue interpretada como secundaria al uso de valproato, por lo que debió modificarse el tratamiento anticonvulsivante. El resto del estudio bioquímico, hematológico y metabólico fue normal.

En Abril del año 2004 se rehospitaliza por un sínd rome de alteración de conciencia. Al ingreso destacaba deterioro cognitivo, con aumento en la frecuencia de estados catatónicos y menor respuesta al tratamiento anticonvulsivante (carbamazepina y clonazepam). Estos estados fueron interpretados como catatonía orgánica. Se realizó nueva TAC que confirmó la atrofia fronto temporal (Fig 2) y un SPECT que evidenciaba hipoperfusión en las mismas regiones (Fig 3). EEG seriados registraron un fondo lento theta de bajo voltaje, de apariencia encefalopática, con paroxismos lentos de mayor amplitud frontotemporal izquierda que tenían tendencia a generalizarse. Pruebas hepáticas, glutamina, anticuerpos antitiroídeos, VDRL y LCR fueron normales descart á ndose otras causas de daño orgánico cerebral.

La evaluación neuropsicológica mostró preservación de la eficiencia cognitiva global y del lenguaje ${ }^{4}$ (Tabla 1). 


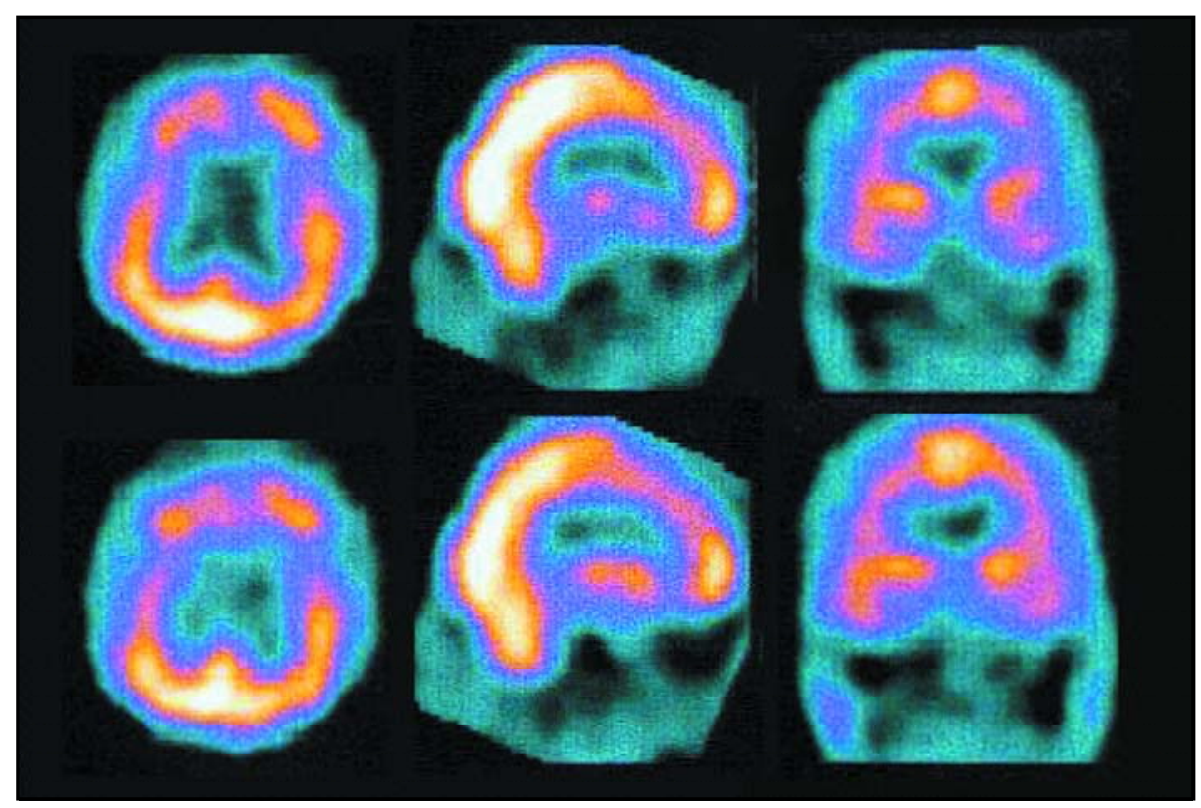

Fig 3. SPECT de encéfalo que evidencia marcada hipoperfusión de los lóbulos frontales y temporales.

Tabla. Evaluación neuropsicológica*.

\begin{tabular}{lc}
\hline Test & Puntaje \\
\hline Eficiencia cognitiva global & 27 \\
$\quad$ Mini-Mental Test de Folstein $(24 \pm 1)$ & \\
Atención y memoria de trabajo & 4 \\
$\quad$ Span directo de dígitos $(6 \pm 1)$ & 4 \\
$\quad$ Span inverso de dígitos $(5 \pm 1)$ & \\
Actividades visoconstructivas & 5 \\
$\quad$ Copia de figuras geométricas simples $(5 \pm 1)$ & 16 \\
$\quad$ Copia de la figura de Rey $(30 \pm 4)$ & 22 \\
Copia de la figura de Rey con programa $(30 \pm 4)$ & 13,5 \\
Copia de la figura de Rey con claves espaciales $(30 \pm 4)$ &
\end{tabular}

Memoria episódica verbal (Test de Grober y Buschke)

Recuerdo inmediato $(15 \pm 0.5) \quad 15$

Recuerdo libre total $(39 \pm 5) \quad 27$

Suma del recuerdo libre y total $(46 \pm 2) \quad 46$

Intrusiones (0) 0

Recuerdo libre diferido $(14 \pm 1.5) \quad 13$

Suma del recuerdo diferido libre y total $(16 \pm 1) \quad 15$

Memoria episódica visual

Test de reconocimiento de caras de Warrington (46 \pm 7) 36

Funciones ejecutivas

Frontal Assessment Battery (FAB) $(15 \pm 1) \quad 14$

Fluencia verbal categorial $(23 \pm 5) \quad 13$

Fluencia verbal morfológica $(16 \pm 5)$

Elaboración conceptual y flexibilidad (Test de clasificación de cartas de Wisconsin)

Categorías $(5 \pm 1)$

Errores perseverativos $(2 \pm 1)$ 12

* Los números entre paréntesis indican el promedio \pm desviación estándar de un grupo control de similar edad y escolaridad que el paciente. 
Las mayores dificultades consistían en un trastorno atencional; un déficit en la capacidad de evocación en memoria episódica, con normalidad de los rendimientos con la introducción de claves facilitadoras; déficits en la copia de la figura compleja de Rey, que mejoraba con la introducción de un programa, aunque no con la introducción de puntos de referencia espaciales y trastornos en las funciones ejecutivas, expresado por una disminución de la fluencia verbal, trastornos de la conceptualización y de la flexibilidad ${ }^{5-7}$.

\section{DISCUSION}

En este paciente destaca la presentación de una fo rma poco común de epilepsia, corroborada con la observación clínica y electroencefalográfica, y de iniciación tardía. Presenta crisis de alteración de conciencia que simulan un estado catatónico de la rga duración, asociado a descargas mioclónicas de la cara. Esta condición mejoraba con el uso de anticonvulsivantes y luego tendía alarefractariedad a pesar del uso de distintos esquemas terapéuticos. En el estudio con neuroimágenes, se observó disminución del volumen encefálico, a expensas de regiones corticales anteriores y frontotemporales, y no se detectaron lesiones focales que pudieran explicar una epilepsia secundaria. Específicamente, en la DFT no familiar no se ha mencionado la presencia de crisis epilépticas como parte de sus fenotipos clínicos. Las demencias hereditarias tienen un inicio temprano, están ligadas a mutaciones en el cromosoma 17 y se asocian habitualmente a parkinsonismo ${ }^{8}$, La epilepsia, como enfermedad puede cursar con distintos niveles de deterioro cognitivo que puede originarse derivado del propio tratamiento con antiepilépticos, ser secundarios al daño hipóxico de las crisis, e incluso desarrollar un deterio rocognitivo transitorio que podría obsenvarse en relación a descargas interictales ${ }^{9}$. La manifestación de fenómenos catatónicos dentro de los síntomas ictales de este paciente nos orienta a un compromiso frontal. Entre las numerosas etiologías n e u rológicas de la catatonía se cuentan enferm edades del lóbulo frontal. Cabe considerar también que el status epiléptico no convulsivo y el síndrome catatónico pueden compartir características clínicas y su diagnóstico diferencial en base al examen físico o la respuesta al uso de benzodiazepinas podría ser muy difícil, quedando sólo el EEG como la herramienta más útil para efectuar esta distinción ${ }^{10}$

En nuestro paciente, como ya fue señalado, no se encontró en la TC cerebral ninguna anormalidad estructural que pudiese explicar una epilepsia tardía secundaria, de modo que la aparición de tras- tornos conductuales y la presencia de daño cognitivo, a g regado a la acentuación del síndrome epiléptico y del daño global nos permite establecer un cuadro sugerente de degeneración frontal y pemitirían hipotetizar un mecanismo etiopatogénico unívoco.

Las características clínicas que han permitido sospechar la disfunción frontal en este paciente han sido el comienzo insidioso antes de los 65 años, su apatía progresiva, la rigidez mental que origin aban descalificaciones injustificadas de sus amistades y conductas estereotipadas como el exagerado interés por la lectura de literatura médica y la compulsión por ejercicios de caminatas.

Los trastornos cognitivos evidenciados en la evaluación neuropsicológica sugieren un compromiso de las regiones fronto-subcorticales con preservación de las regiones postrolándicas. En efecto, la normalización del recuerdo en el test de GroberBuschke con la introducción de claves semánticas p e rmite concluir que las dificultades pueden atribuirse particula mente a una disfunción de los circuitos fronto-subcorticales y no al de las regiones hipocampales ${ }^{11}$. Del mismo modo, la mejoría obsenada en la copia de la figura de Rey, con la adición de un programa, permiten concluir que los trastornos visoconstructivos serían secundarios a un trastorno de las funciones ejecutivas y no relacionados con las funciones espaciales dependientes de las regiones parietales ${ }^{7}$. En suma, el perfil observado en los trastornos cognitivos, donde lo que predomina es una alteración de las funciones ejecutivas suelen ser característicos de las demencias frontotemporales.

Los exámenes de laboratorio de rutina, como era esperable en una demencia degenerativa resultaron normales. Aunque el EEG en la DFT suele ser normal, en este caso se observó sobre una lentitud generalizada del trazado, actividad irritativa sobreimpuesta que definen una epilepsia concomitante.

Las neuroimágenes apoyaron el diagnóstico de degeneración frontal. En las tomografías computadas se encontró atrofia cortical moderada, predominantemente frontotemporal y en lo funcional, el SPECT evidenciaba una categórica hipoperfusión frontal bilateral .

La asociación de demencia y epilepsia obliga a pensar en diagnósticos diferenciales, tales como porfiria aguda intermitente, neuroacantocitosis, déficit de vitamina B12, enfermedad de Lyme y encefalopatía de Hashimoto, condiciones que en nuestropaciente fueron descartadas tanto por los 
atributos clínicos como por los exámenes correspondientes.

El tratamiento de la epilepsia fue eficaz en los primeros años con fenitoína y valproato. Sin emb a rgo, en su última hospitalización debió modificarse el esquema introduciéndose carbamazepina $600 \mathrm{mg} / \mathrm{día}$, debido a la insuficiente respuesta clínica y a la progresión de los síntomas.

El manejo de la DFT es pobre. No hay terapias que puedan prevenir la progresión de la enfermedad y existe una impresión clínica de que los anticolinesterásicos no producen mejoría sintomática e incluso pueden aumentar los trastornos conductuales. Recientemente se ha publicado un pequeño estudio doble ciego, controlado con placebo, donde trazodone redujo en un $50 \%$ el score del Neuro Psychiatric Inventory en la mitad de los pacientes, con mejoría principalmente en irritabilidad, agitación, síntomas depresivos y trastornos del comer ${ }^{12}$ Hasta ahora, no ha sido comunicada la asociación que motiva la presentación del caso. Parece coherente que un proceso etiopatogénico común haya expresado primero una forma inusual de epilepsia generalizada y posteriormente la instalación de una demencia de tipo frontotemporal. No podemos descartarla opción de una asociación incidental y secuencial de estas dos entidades, sólo definible con el consecuente estudio neuropatológico.

\section{REFERENCIAS}

1. Neary D, Snowden JS, Gustafson L, et al. Frontotemporal lobar degeneration: a consensus on clinical diagnostic criteria. Neurology 1998; 51:1546-1554

2. Foster NL, Wilhelmsen K, Sima AA., Jones MZ, D'Amato CJ,Gilman S. Frontotemporal dementia and parkinsonism linked to chromosome 17: a consensus conference. Ann Neurol 1997;41:706-715.

3. Neary D, Snowden JS, Mann DM. The clinical pathological correlates of lobar atrophy. Dementia 1993;4:154-159.

4. Folstein MF, Folstein SE, McHugh PR. Mini-mental state: a practical method for grading the cognitive state of patients for the clinician. J Psychiatry Res 1975;12:189-198.

5. Grober E, Buschke H, Crystal H, Bang S, Dresner R. Screening for dementia by memory testing. Neurology 1988;38:900-903.

6. Nelson HE. A modified card sorting test sensitive to frontal lobe defects. Cortex 1976;12:313-324.

7. Pillon B. Troubles visuo-constructifs et méthodes de compensation résultats de 85 patients atteints de lésions cérebrales. Neuropsychologia 1981;19:375-383.

8. Sperfeld AD, Collatz MB, Baier H, et al. FTDP-17: an early-onset phenotype with parkinsonism and epileptic seizures caused by a novel mutation. Ann Neurol 1999;46:708-715.

9. Aarts JH, Binnie CD, Smit AM, Wilkins AJ. Selective cognitive impairment during focal and generalized epileptiform EEG activity. Brain 1984;107(Pt 1):293-308.

10. Louis ED, Pflaster NL. Catatonia mimicking nonconvulsive status epilepticus. Epilepsia 1995;36:943-945.

11. Pillon B, Dubois B, Lhermitte F, Agid Y. Heterogeneity of cognitive impairment in progressive supranuclear palsy, Parkinson's disease, and Alzheimer's disease. Neurology 1986;36:1179-1185.

12. Lebert F, Pasquier F, Souliez F, Petit H. Frontotemporal behavioral scale Alzheimer Dis Assoc Disord 1998;12:335-339. 